University of New Hampshire

University of New Hampshire Scholars' Repository

Space Science Center

Institute for the Study of Earth, Oceans, and

Space (EOS)

1994

\title{
Tracking and imaging gamma ray experiment (TIGRE) for 1 to 100 MEV gamma ray astronomy
}

\author{
A Alpar \\ Middle East Technical University \\ D Bhattacharya \\ University of California - Riverside \\ R Buccheri \\ IFCAI \\ $\mathrm{K}$ Dotson \\ NOVA R\&D Inc. \\ D J. Forrest \\ University of New Hampshire - Main Campus

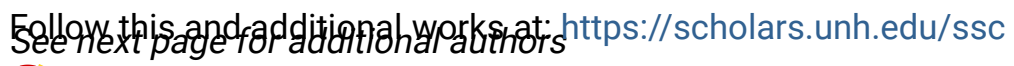 \\ Part of the Astrophysics and Astronomy Commons
}

\section{Recommended Citation}

Tracking and imaging gamma ray experiment (TIGRE) for 1 to 100 MEV gamma ray astronomy Alpar, A. and Bhattacharya, D. and Buccheri, R. and Dotson, K. and Forrest, D. and Johnson, W. N. and Kanbach, G. and Kiziloglu, U. and Kroeger, R. and Kurfess, J. and McConnell, M. and Ögelman, H. and O'Neill, B. and O'Neill, T. and Owens, A. and Pi, B. and Pierce, B. and Ryan, J. and Sacco, B. and Simnett, G. and Tümer, T. and Wheaton, W. and White, R. S. and Zych, A., AIP Conference Proceedings, 304, 701-705 (1994), DOI:http://dx.doi.org/10.1063/1.45559

This Conference Proceeding is brought to you for free and open access by the Institute for the Study of Earth, Oceans, and Space (EOS) at University of New Hampshire Scholars' Repository. It has been accepted for inclusion in Space Science Center by an authorized administrator of University of New Hampshire Scholars' Repository. For more information, please contact Scholarly.Communication@unh.edu. 
Authors

A Alpar, D Bhattacharya, R Buccheri, K Dotson, D J. Forrest, W N. Johnson, G Kanbach, U Kiziloglu, R Kroeger, J Kurfess, M L. McConnell, H Ogelman, B O'Neill, Terence J. O'Neill, A Owens, B Pi, B Pierce, James M. Ryan, B Sacco, G Simnett, O T. Tumer, W Wheaton, R S. White, and Allen Zych 


\section{AIP $\mid$ proceedings}

\section{Tracking and imaging gamma ray experiment (TIGRE) for 1 to $100 \mathrm{MEV}$ gamma ray astronomy}

A. Alpar, D. Bhattacharya, R. Buccheri, K. Dotson, D. Forrest, W. N. Johnson, G. Kanbach, U. Kiziloglu, R. Kroeger, J. Kurfess, M. McConnell, H. Ögelman, B. O'Neill, T. O'Neill, A. Owens, B. Pi, B. Pierce, J. Ryan, B. Sacco, G. Simnett, T. Tümer, W. Wheaton, R. S. White, and A. Zych

Citation: AIP Conference Proceedings 304, 701 (1994); doi: 10.1063/1.45559

View online: $\mathrm{http}: / / \mathrm{dx}$. doi.org/10.1063/1.45559

View Table of Contents:

http://scitation.aip.org/content/aip/proceeding/aipcp/304?ver=pdfcov

Published by the AIP Publishing

Articles you may be interested in

Minute-of-Arc Resolution Gamma ray Imaging Experiment-MARGIE AIP Conf. Proc. 510, 779 (2000); 10.1063/1.1303304

COMPTEL measurements of $1.809 \mathrm{MeV}$ gammaray line emission from the Galactic plane

AIP Conf. Proc. 280, 40 (1993); 10.1063/1.44309

COMPTEL observations of cosmic gammaray bursts

AIP Conf. Proc. 265, 22 (1991); 10.1063/1.42837

A new method to search for high energy gamma rays by trackcalorimeter detectors at a high mountain

AIP Conf. Proc. 220, 334 (1991); 10.1063/1.40319

Imaging germanium telescope array for gammarays (IGETAGRAY)

AIP Conf. Proc. 211, 224 (1990); 10.1063/1.39687 


\title{
TRACKING AND IMAGING GAMMA RAY EXPERIMENT (TIGRE) FOR 1 TO 100 MEV GAMMA RAY ASTRONOMY
}

\author{
A. Alpar ${ }^{1}$, D. Bhattacharya ${ }^{2}$, R. Buccheri ${ }^{3}$, K. Dotson ${ }^{4}$, D. Forrest ${ }^{5}$, W. N. Johnson ${ }^{6}$ G. \\ Kanbach $^{7}$, U. Kiziloglu, R. Kroeger ${ }^{6}$, J. Kurfess ${ }^{6}$, M. McConnell ${ }^{5}$, H. Ögelman ${ }^{8}$, B.

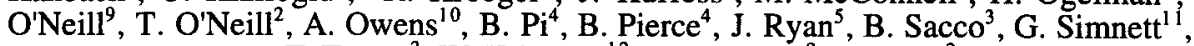 \\ T. Tümer ${ }^{2}$, W. Wheaton ${ }^{12}$, R. S. White ${ }^{2}$ A. Zych ${ }^{2}$ \\ ${ }^{1}$ Middle East Technical University, Turkey, ${ }^{2}$ University of California, Riverside, ${ }^{3}$ IFCAI, \\ Italy, ${ }^{4}$ NOVA R\&D Inc., Riverside, CA,${ }^{5}$ University of New Hampshire, ${ }^{6}$ Naval Research \\ Laboratory, ${ }^{7}$ MPE, Germany, ${ }^{8}$ University of Wisconsin, ${ }^{9}$ University of Redlands, ${ }^{10}$ \\ University of Leicester, UK, ${ }^{11}$ University of Birmingham, UK, ${ }^{12}$ Jet Propulsion Laboratory
}

\section{ABSTRACT}

A large international collaboration from the high energy astrophysics community has proposed the Tracking and Imaging Gamma Ray Experiment (TIGRE) for future space observations. TIGRE will image and perform energy spectroscopy measurements on celestial sources of gamma rays in the energy range from 1 to $100 \mathrm{MeV}$. This has been a difficult energy range experimentally for gamma ray astronomy but is vital for the future considering the recent exciting measurements below 1 and above $100 \mathrm{MeV}$. TIGRE is both a double scatter Compton and gamma ray pair telescope with direct imaging of individual gamma ray events. Multi-layers of Si strip detectors are used as Compton and pair converters and $\mathrm{CsI}(\mathrm{Tl})$ scintillation detectors are used as a position sensitive calorimeter. Alternatively, thick Ge strip detectors may be used for the calorimeter. The Si detectors are able to track electrons and positrons through successive Si layers and measure their directions and energy losses. Compton and pair events are completely reconstructed allowing each event to be imaged on the sky. TIGRE will provide an order-of-magnitude improvement in discrete source sensitivity in the 1 to $100 \mathrm{MeV}$ energy range and determine spectra with excellent energy and excellent angular resolutions. It's wide field-of-view of $\pi$ sr permits observations of the entire sky for extended periods of time over the life of the mission.

\section{INTRODUCTION}

The Compton Gamma Ray Observatory (CGRO) is now observing the gamma ray sky over a range of more than six decades in energy $\left(\sim 10^{-2}-3 \times 10^{4} \mathrm{MeV}\right)$. The results from CGRO are presently redefining the field of high energy astrophysics in a manner that will extend into the $21^{\text {st }}$ century. It is clear that multi-wavelength and multi-epoch measurements will be needed in the foreseeable future. At the same time it is imperative to identify the gaps in the energy range which are not fully being covered by the CGRO. This calls for the active development of new instruments with improved resolutions and sensitivities to supplement the CGRO observations. In this paper we describe an improved electron tracking telescope for future space observations. The scientific goals of the Tracking and Imaging Gamma Ray Experiment (TIGRE) will be to accurately image with few arc minute resolution and derive energy spectra of cosmic discrete and diffuse gamma ray sources in the (1-100) MeV range.

The gamma ray detection techniques in the intermediate energy range of (1-100) MeV belong to the Compton and pair production processes in photon-matter interactions. At about $10 \mathrm{MeV}$, the pair production cross-section starts to dominate. Hence, the sensitivity of instruments employing a singular technique to register gamma ray photons changes dramatically over this energy range. The COMPTEL effective area decreases above $10 \mathrm{MeV}$, whereas the EGRET effective area falls rapidly below $100 \mathrm{MeV}$. Hence, in the intermediate energy range of 10 to $100 \mathrm{MeV}$ not many sources have been discovered and the behavior of many pulsars 
or AGNs, seen in low or high energies, remains unknown. TIGRE is designed to operate in the difficult intermediate energy range which includes the low $\mathrm{MeV}$ nuclear line region as well as the high energy range that now appears to be dominated by "Blazar" QSOs and BL Lac objects.

The energy range (1-100) MeV is important because it contains the critical signatures of a variety of emission processes, including electron bremsstrahlung, cosmic-ray/matter interactions, synchrotron self-Compton processes, etc. A proper evaluation of the gamma ray spectral shape in this range is necessary because the results of OSSE, COMPTEL and EGRET indicate that the emission spectra of several AGN and pulsars may contain breaks in this energy range. Furthermore, the absence of sensitive measurements with good angular resolution have failed to produce the predicted number of $\mathrm{MeV}$ sources. The goal of the TIGRE mission will be to obtain the medium $\mathrm{MeV}$ spectra of different types of galactic and extragalactic discrete sources in order to identify their radiation mechanisms and explain their episodic behavior as well as to spatially resolve MeV sources and map the diffuse galactic emission.

\section{INSTRUMENT DESCRIPTION}

TIGRE (Figure 1) merges two new technologies in radiation detection: 1) large area wafer-thin silicon strip detectors (SSD) which have proved so successful in tracking minimum ionizing particles in high energy accelerator experiments and 2) CsI(T1) scintillation crystals coupled to large area photodiode detectors for improved energy resolution. TIGRE uses the double scatter technique with an active tracking converter of silicon strip detectors and an energy absorbing position sensitive calorimeter of CsI(Tl)-photodiode detectors to detect incident gamma rays over the wide energy range of $1-100 \mathrm{MeV}$. Incident directions and energies are measured directly and uniquely with high resolutions. As an option, the CsI(Tl)-photodiode position sensitive calorimeter can be replaced with thick Germanium strip detectors which are now being developed (Gutknecht 1990). The further improvements in performance of TIGRE with this option will be indicated where appropriate. TIGRE is also a gamma ray polarimeter, making use of the sensitivity of the Kline-Nishina cross sections for large angle Compton scatter events.

The incident gamma ray Compton scatters in a single layer of an array of doublesided $300 \mu \mathrm{m}$ thick silicon strip detectors.

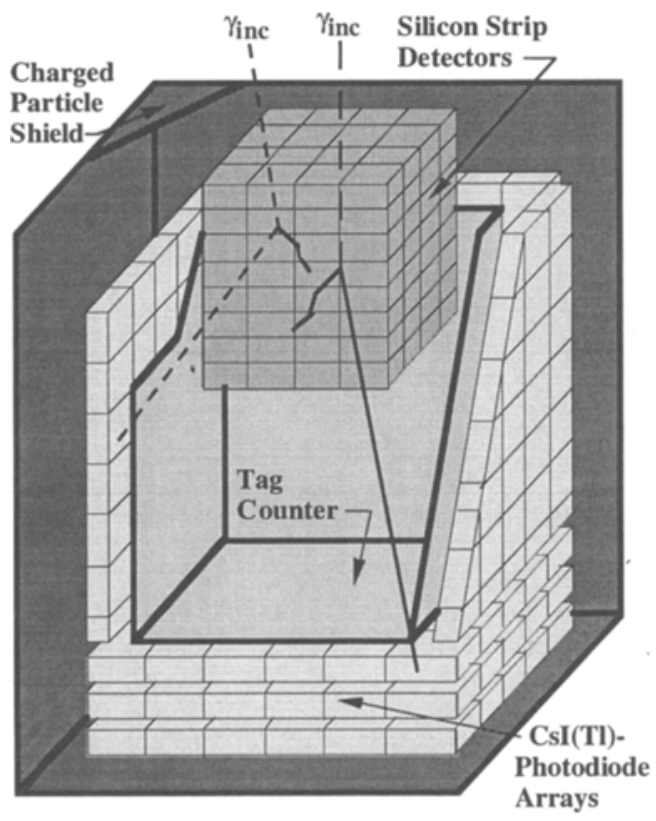

Figure 1. Tracking and Imaging Gamma Ray Experiment (TIGRE)

The Compton recoil electron is tracked through successive SSD layers until it is fully absorbed. The electron energy loss in each layer is measured to a resolution of $9 \mathrm{keV}$ (FWHM). The initial direction of the recoil electron is determined from the positions in the layer of the initial interaction and the adjacent layer. The layers are $5 \mathrm{~mm}$ apart. The $\mathrm{x}$ - and $\mathrm{y}$-positions of the electron traversing each layer are determined to an accuracy of $0.8 \mathrm{~mm}$, the pitch of the orthogonal readout strips. These positions allow the initial direction of the recoil electron to be determined to about $10^{\circ}$, comparable to the multiple scattering of a $3 \mathrm{MeV}$ electron in a 
single SSD layer. The direction and energy of the Compton scattered gamma ray are measured with an array of CsI(Tl) scintillation detectors coupled to photodiodes. TIGRE, with recoil electron tracking, gives an unique incident direction for each Compton event so that the source can be directly imaged. We have achieved energy resolutions of $5 \%$ at $662 \mathrm{keV}$ for 1 $\mathrm{cm} \times 1 \mathrm{~cm} \times 2.5 \mathrm{~cm}$ CsI(Tl) crystals coupled to photodiodes. Using Germanium strip detectors as an alternative to the CsI(Tl)-photodiode detectors with their $1 \mathrm{~mm}$ spatial and 2 $\mathrm{keV}$ (FWHM) energy resolutions gives an outstanding gamma ray instrument with unsurpassed performance.

Pair events are detected in the traditional manner by tracking individually the electron and positron through successive layers of silicon strip detectors until they are either fully absorbed in the silicon or they exit the SSD and interact in the CsI(Tl) calorimeter. Both the energy losses and positions of the pair particles are measured in each SSD layer as these particles are tracked through the array. The incident gamma ray direction is reconstructed from the tracking information. It's energy is estimated from the total energy losses in the SSD and CsI(Tl) detectors.

The silicon strip detector array consists of 80 layers of silicon strip detectors each separated by $5 \mathrm{~mm}$. Each layer has 16 individual detectors of dimensions $10 \mathrm{~cm} \mathrm{x} 10 \mathrm{~cm} \mathrm{x}$ $300 \mu \mathrm{m}$ thick mounted adjacent to each other in a $4 \times 4$ matrix. Each layer has a sensitive area of $1,600 \mathrm{~cm}^{2}$. The total thickness of the silicon is $5.8 \mathrm{~g} \mathrm{~cm}^{-2}$. Each silicon detector side has 128 readout strips with $0.8 \mathrm{~mm}$ pitch on the junction side and 128 orthogonal strips on the ohmic side.

Small CsI(Tl) scintillation crystals $(1 \mathrm{~cm} \mathrm{x} 1 \mathrm{~cm} \times 2.5 \mathrm{~cm})$ coupled to large area $1 \mathrm{~cm}$ $x 1 \mathrm{~cm}$ silicon PIN diode detectors offer higher material density $\left(4.51 \mathrm{gm} \mathrm{cm}^{-3}\right)$ and improved energy resolution. Three CsI(Tl)-photodiode detector assemblies with sensitive areas of $2,304 \mathrm{~cm}^{2}$ are placed below the silicon array at an average distance of $50 \mathrm{~cm}$. Each assembly is $2.5 \mathrm{~cm}$ thick for a total crystal thickness of $33.8 \mathrm{~g} \mathrm{~cm}^{-2}$. An equivalent thickness for Germanium $\left(5.32 \mathrm{~g} \mathrm{~cm}^{-3}\right)$ is $6 \mathrm{~cm}$. These detectors measure the directions of the scattered gamma rays from the silicon array to an accuracy of $<1^{0}$ for $\mathrm{CsI}$ and $<0.1^{\circ}$ for $\mathrm{Ge}$.

Four CsI(TI) assemblies are positioned on the sides of the silicon array. These side assemblies detect Compton scattered gamma rays with large scatter angles and pair events with large opening angles.

The entire TIGRE instrument is enclosed in a charged particle anticoincidence shield of thin $(0.635 \mathrm{~cm})$ plastic scintillator. We have also introduced a thin $(0.635 \mathrm{~cm})$ plastic scintillator tag counter to indicate the passage of a charged particle from the silicon to the CsI(Tl). This will be important for the diagnostics of gamma ray pair events in the silicon and for identifying recoil electrons that leave the SSD layers.

\section{MONTE CARLO SIMULATIONS}

A comprehensive model of the prototype TIGRE instrument has been inserted into the general purpose MCNP (Monte Carlo Neutron Photon) code developed at Los Alamos. The model includes 50 layers of silicon strip detectors with $5 \mathrm{~mm}$ spacing. Each layer consists of a $12.8 \mathrm{~cm} \times 12.8 \mathrm{~cm} \times 300 \mu \mathrm{m}$ thick SSD with $1 \mathrm{~mm}$ pitch. Each layer has a perimeter support structure of PCB. Over 10,000 individual $1 \mathrm{~cm}^{2}$ CsI(Tl) scintillation crystals surround the SSD stack on five sides. Tag counters are placed between the SSD and CsI(Tl) and the entire instrument is surrounded by a plastic scintillator veto counter. Complete Monte Carlo histories were generated for gamma rays incident on the detector at 1, 2, 6, 10, 25, 50, 75 and $100 \mathrm{MeV}$. Both Compton and pair events were identified and recorded. Energy and spatial resolutions were included in all the simulated measurements. At $1 \mathrm{MeV}$, about $68 \%$ of the double scatter events have recoil electrons traversing at least 2 SSD layers. At $2 \mathrm{MeV}$ it is about $88 \%$. These are the "tracked events" for which an unique incident direction can be assigned to the detected gamma ray. Equal numbers of Compton and pair 


\section{TIGRE}

events are detected at about $15 \mathrm{MeV}$. The energy spectra for $1 \mathrm{MeV}$ and $100 \mathrm{MeV}$ normal incident gamma rays are shown in Figures $2 \mathrm{a}$ and $2 \mathrm{~b}$. The effect of the energy loss in the SSD support structure is evident in Figure $2 b$.

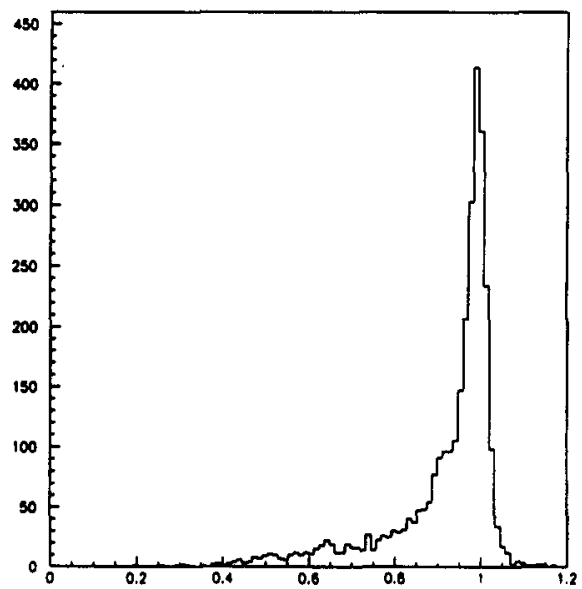

(a)

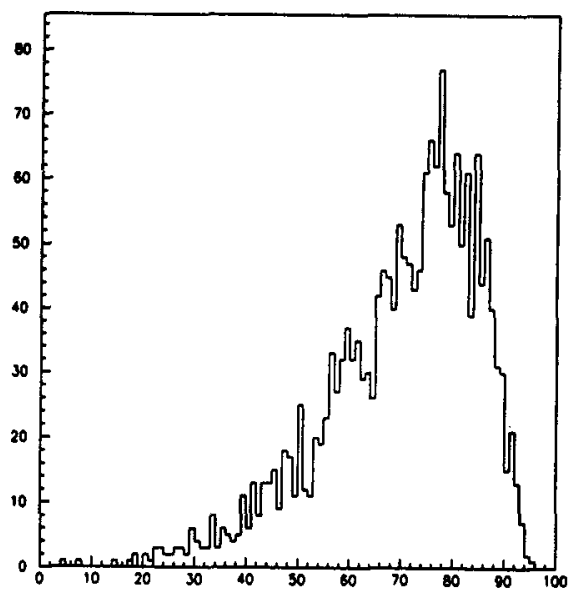

(b)

Figure 2. Total detected energy loss for a) $1 \mathrm{MeV}$ and b) $100 \mathrm{MeV}$ incident photons

The simulated energy and angular resolutions for Compton events are shown in Figure 3. For Compton telescopes, the angular resolution reflects the width of the event annulus on the sky when recoil electron tracking is not used. It is dependent on both the energy and spatial resolutions individual detectors. The improvement with $\mathrm{Ge}$ is also shown here.
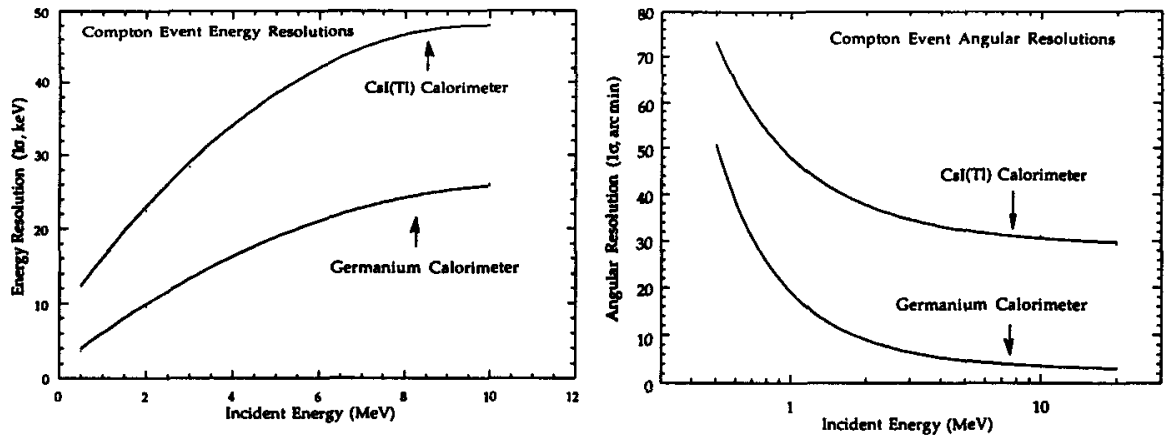

Figure 3. TIGRE energy and angular resolutions for Compton events.

The absolute detection efficiency of TIGRE as a function of incident energy is shown in Figure 4. This efficiency includes Compton events only where the recoil electron can be tracked in the Si. The maximum efficiency, at $25 \mathrm{MeV}$, is $8.2 \%$. This high efficiency is due to the contribution of the side calorimeters. This efficiency decreases by only $60 \%$ at an incident angle of $60^{\circ}$ to give a large FOV of $\pi$ sr. The maximum efficiency corresponds to an effective area of $130 \mathrm{~cm}^{2}$ for a SSD area of $1,600 \mathrm{~cm}^{2}$. These calculations show that Compton scattering and pair production combine to give a gamma ray telescope with essentially constant response from 1 to $100 \mathrm{MeV}$.

Both Compton and pair events can be directly imaged. For the former, recoil electron 
tracking allows an unique incident direction to be determined for each event, making imaging relatively easy. Figure 5 compares the images for $25 \mathrm{MeV}$ for Compton (left) and pair (right) events. The pair image is broader due to the relatively large opening angle for the electronpositron pair.
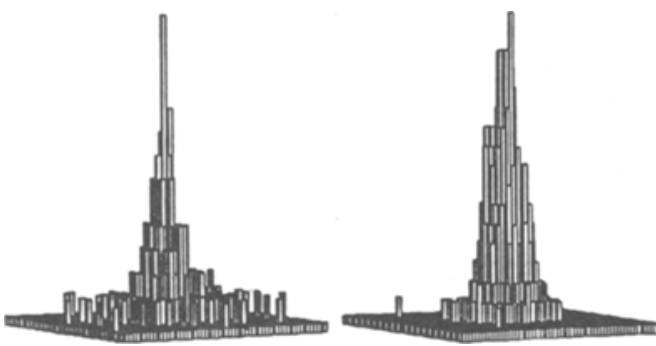

Figure 5. Compton (left) and Pair Images (right) at $25 \mathrm{MeV}$.

The sensitivity for observing continuum and gamma ray line emissions from discrete sources is based on TIGRE's imaging properties and energy resolution. We have taken an exposure time of $2.4 \times 10^{6}$ seconds, a 28 day period with $100 \%$ duty cycle. The limiting gamma ray background is taken as the isotropic cosmic diffuse flux. The $3 \sigma$ sensitivities are shown to the right in Figure 6. With Ge strip detectors used as the calorimeter the continuum sensitivity (not shown) is about a factor of 2 lower.

\section{CONCLUSIONS}

We believe that the Tracking and Imaging Gamma Ray Experiment described here has the potential to make very high sensitivity observations from 1 to $100 \mathrm{MeV}$ by combining the Compton and pair detection in a single instrument. Electron tracking with silicon strip detectors provides a new technique to be exploited in gamma ray astronomy.
Figure 4. TIGRE Absolute Efriency
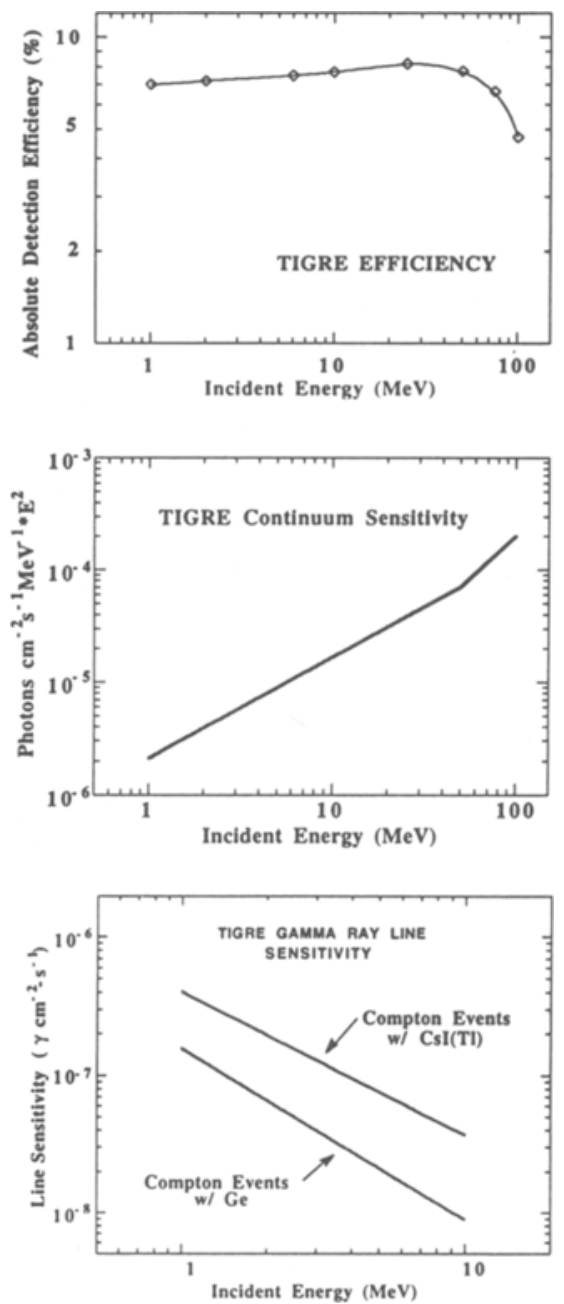

Figure 6. TIGRE Sensitivities

We wish to acknowledge the support of NASA grant NAGW 1996.

\section{REFERENCES}

Gutknecht, D., 1990, Nuc. Inst. And Meth. A228, 13. 